\title{
Well-posedness in Maxwell systems with distributions of polarization relaxation parameters
}

\author{
H.T. Banks*, N.L. Gibson \\ Center for Research in Scientific Computation, North Carolina State University, Raleigh, NC 27695-8205, United States
}

Received 1 January 2004; accepted 1 February 2004

\begin{abstract}
We present existence, uniqueness and continuous dependence (with respect to probability distributions on polarization parameters) of solutions in Maxwell systems. This provides a theoretical and computational foundation for associated inverse problems.

(C) 2004 Elsevier Ltd. All rights reserved.

Keywords: Well-posedness; Maxwell's equations with polarization; Distributed relaxation parameters
\end{abstract}

\section{Introduction}

In this note we consider well-posedness questions for the variational solutions of one dimensional Maxwell's equations with an absorbing left boundary condition, a supraconducting right boundary condition and a general macroscopic polarization term which includes uncertainty in the dielectric parameters. For these solutions, we establish existence, uniqueness and continuous dependence on the uncertainty measures in a Prohorov metric (see [4,1] for definitions and details) sense. As explained below, these results can be readily used in an inverse problem methodology to determine the unknown distribution of the dielectric parameters which govern the behavior of the electric field and the electric polarization in a general heterogeneous material with multiple mechanisms (Debye, Lorentz, etc.) and relaxation parameters.

\footnotetext{
* Corresponding address: Center for Research in Scientific Computation, Department of Mathematics, North Carolina State University, Box 8205, Raleigh, NC 27695-8205, United States. Tel.: +1 9195153968.

E-mail addresses: htbanks@ unity.ncsu.edu (H.T. Banks), ngibson@math.ncsu.edu (N.L. Gibson).
} 


\section{Problem formulation}

We consider the one dimensional problem formulation in [3]. Assuming $D=\epsilon E+P$, we may obtain Maxwell's equations [6] in second order form given by:

$$
\mu_{0} \epsilon \ddot{E}+\mu_{0} I_{\Omega} \ddot{P}+\mu_{0} \sigma \dot{E}-E^{\prime \prime}=-\mu_{0} \dot{J}_{s} \text { in } \Omega \cup \Omega_{0},
$$

where $E$ is the transverse component of the electric field, $P$ is the material macroscopic electric polarization, $\epsilon=\epsilon(z)$ is the dielectric permittivity and $\sigma=\sigma(z)$ is the conductivity of the material. The boundary conditions that we are assuming are absorbing at $z=0$ and supraconducting at $z=1$ :

$$
\left[\dot{E}-c E^{\prime}\right]_{z=0}=0, \quad E(t, 1)=0 .
$$

Our initial conditions are

$$
E(0, z)=\Phi(z), \quad \dot{E}(0, z)=\Psi(z) .
$$

To describe the behavior of the electric polarization $P$, we begin with the general formulation of Chapter 2 of [3] by employing a polarization kernel $g$ in the convolution expression

$$
P(t, z)=\int_{0}^{t} g(t-s, z ; \tau) E(s, z) \mathrm{d} s .
$$

As explained in [3], this general formulation includes as special cases the well known orientational or Debye polarization model, the electronic or Lorentz polarization model, and linear combinations thereof, as well as other higher order models. In the Debye case the kernel is given by

$$
g(t ; \tau)=\left(\epsilon_{0}\left(\epsilon_{s}-\epsilon_{\infty}\right) / \tau\right) \mathrm{e}^{-t / \tau},
$$

while in the Lorentz model (again see [3]), it takes the form

$$
g(t ; \tau)=\epsilon_{0} \omega_{p}^{2} / \nu_{0} \mathrm{e}^{-t / 2 \tau} \sin \left(v_{0} t\right) .
$$

However, use of these kernels presupposes that the material may be sufficiently defined by a single relaxation parameter $\tau$, which is generally not the case. In order to account for multiple relaxation parameters in the polarization mechanisms, we allow for a distribution of relaxation parameters which is conveniently described in terms of a probability measure $F$. Thus, we define our polarization model in terms of a convolution operator

$$
P(t, z)=\int_{0}^{t} \mathcal{G}(t-s, z) E(s, z) \mathrm{d} s,
$$

where $\mathcal{G}$ is determined by various polarization mechanisms each described by a different parameter $\tau$, and therefore is given by

$$
\mathcal{G}(t, z ; F)=\int_{\mathcal{T}} g(t, z ; \tau) \mathrm{d} F(\tau),
$$

where $\mathcal{T} \subset\left[\tau_{1}, \tau_{2}\right]$. In particular, if the distribution were discrete, consisting of a single relaxation parameter, then we would again have (4).

Note that,

$$
\ddot{P}(t, z)=\int_{0}^{t} \ddot{\mathcal{G}}(t-s, z) E(s, z) \mathrm{d} s+\mathcal{G}(0, z) \dot{E}(t, z)+\dot{\mathcal{G}}(0, z) E(t, z),
$$


where

$$
\ddot{\mathcal{G}}(t, z ; F)=\int_{\mathcal{T}} \ddot{g}(t, z ; \tau) \mathrm{d} F(\tau) .
$$

Substituting (5) into (1) we obtain

$$
\begin{gathered}
\mu_{0} \epsilon \ddot{E}(t, z)+\mu_{0} I_{\Omega}[\sigma+\mathcal{G}(0, z)] \dot{E}(t, z)+\mu_{0} I_{\Omega} \dot{\mathcal{G}}(0, z) E(t, z) \\
+\int_{0}^{t} \mu_{0} I_{\Omega} \ddot{\mathcal{G}}(t-s, z) E(s, z) \mathrm{d} s-E^{\prime \prime}(t, z)=-\mu_{0} \dot{J}_{s}(t, z) .
\end{gathered}
$$

Further, converting to weak form (and multiplying both sides by $c^{2}$ ), we find

$$
\begin{aligned}
& \left\langle\tilde{\epsilon_{r}} \ddot{E}(t, \cdot), \phi\right\rangle+\left\langle\frac{1}{\epsilon_{0}} I_{\Omega}[\sigma+\mathcal{G}(0, \cdot)] \dot{E}(t, \cdot), \phi\right\rangle+\left\langle\frac{1}{\epsilon_{0}} I_{\Omega} \dot{\mathcal{G}}(0, \cdot) E(t, \cdot), \phi\right\rangle \\
& \quad+\left\langle\frac{1}{\epsilon_{0}} \int_{0}^{t} I_{\Omega} \ddot{\mathcal{G}}(t-s, \cdot) E(s, \cdot) \mathrm{d} s, \phi\right\rangle-\left\langle c^{2} E^{\prime \prime}(t, \cdot), \phi\right\rangle=-\left\langle\frac{1}{\epsilon_{0}} \dot{J}_{s}(t, \cdot), \phi\right\rangle,
\end{aligned}
$$

where $\tilde{\epsilon}_{r}=\epsilon / \epsilon_{0}$ and $\phi \in V=H_{R}^{1}(0,1)=\left\{\phi \in H^{1}(0,1): \phi(1)=0\right\}$. (Without loss of generality, we will hereafter assume $\tilde{\epsilon_{r}}=1$.) Finally, we integrate by parts, and apply the boundary conditions (2) to obtain

$$
\begin{aligned}
& \langle\ddot{E}(t, \cdot), \phi\rangle+\left\langle\frac{1}{\epsilon_{0}} I_{\Omega}[\sigma+\mathcal{G}(0, z)] \dot{E}(t, \cdot), \phi\right\rangle+\left\langle\frac{1}{\epsilon_{0}} I_{\Omega} \dot{\mathcal{G}}(0, \cdot) E(t, \cdot), \phi\right\rangle \\
& \quad+\left\langle\frac{1}{\epsilon_{0}} \int_{0}^{t} I_{\Omega} \ddot{\mathcal{G}}(t-s, \cdot) E(s, \cdot) \mathrm{d} s, \phi\right\rangle+\left\langle c^{2} E^{\prime}(t, \cdot), \phi^{\prime}\right\rangle+c \dot{E}(t, 0) \phi(0)=-\left\langle\frac{1}{\epsilon_{0}} \dot{J}_{s}(t, \cdot), \phi\right\rangle,
\end{aligned}
$$

which we can rewrite as

$$
\begin{aligned}
& \langle\ddot{E}, \phi\rangle+\langle\gamma \dot{E}, \phi\rangle+\langle\beta E, \phi\rangle+\left\langle\int_{0}^{t} \alpha(t-s, \cdot) E(s, \cdot) \mathrm{d} s, \phi\right\rangle+\left\langle c^{2} E^{\prime}, \phi^{\prime}\right\rangle+c \dot{E}(t, 0) \phi(0) \\
& \quad=\langle\mathcal{J}, \phi\rangle, \quad \phi \in V
\end{aligned}
$$

where

$$
\begin{aligned}
& \gamma(z)=\frac{1}{\epsilon_{0}} I_{\Omega}[\sigma(z)+\mathcal{G}(0, z)]=\frac{1}{\epsilon_{0}} I_{\Omega}\left[\sigma(z)+\int_{\mathcal{T}} g(0, z ; \tau) \mathrm{d} F(\tau)\right] \\
& \beta(z)=\frac{1}{\epsilon_{0}} I_{\Omega} \dot{\mathcal{G}}(0, z)=\frac{1}{\epsilon_{0}} I_{\Omega} \int_{\mathcal{T}} \dot{g}(0, z ; \tau) \mathrm{d} F(\tau) \\
& \alpha(t, z)=\frac{1}{\epsilon_{0}} I_{\Omega} \ddot{\mathcal{G}}(t, z)=\frac{1}{\epsilon_{0}} I_{\Omega} \int_{\mathcal{T}} \ddot{g}(t, z ; \tau) \mathrm{d} F(\tau) \\
& \mathcal{J}(t, z)=-\frac{1}{\epsilon_{0}} \dot{J}_{s}(t, z) .
\end{aligned}
$$

\section{Estimation methodology}

Our goal is to estimate the probability distribution function (PDF) of relaxation parameters $F \in$ $\mathcal{P}(\mathcal{T})$ in a given model of the polarization, where $\mathcal{P}(\mathcal{T})$ is the set of all PDFs on the admissible region $\mathcal{T} \subset\left[\tau_{1}, \tau_{2}\right]$. To this end we attempt to minimize the difference between model 
simulations and observations of time-domain data. In our formulation (for details, see [3]), the observations, $\hat{E}_{j}$, are of the electric field $E$ at discrete times $t_{j}$ taken at $z=0$. Each simulation is a solution of Maxwell's equation given in (6) with (3) using candidate values for the distribution of relaxation parameters. We propose a standard least-squares criterion (which is equivalent to a maximum likelihood estimation in many situations; see [5]) for the optimization procedure given by

$$
J(F)=\sum_{j}\left|E\left(t_{j}, 0 ; F\right)-\hat{E}_{j}\right|^{2},
$$

where $E(\cdot, \cdot ; F)$ is the solution of (6) with (3) corresponding to the distribution $F$. Thus the inverse problem is to solve

$$
\min _{F \in \mathcal{P}(\mathcal{T})} J(F)
$$

In practice, one may choose to approximate a continuous distribution $F$ by a discrete one with, for example, $N$ elements. This approach would result in a straight-forward $N$-dimensional minimization problem. Other parameterizations of the unknown distribution are also possible to reduce the problem to a finite dimensional one.

\section{Well-posedness}

In this section we address the questions of well-posedness of the form of Maxwell's equation given in (6) with respect to the unknown distribution of dielectric parameters. In particular we wish to establish the continuous dependence of solutions on the distributions in the sense of the Prohorov metric. This leads in turn to well-posedness for the inverse problems involving (7).

First, given that we assume $g, \dot{g}$, and $\ddot{g}$ are uniformly continuous in $\tau$ and bounded on $([0, T] \times[0,1] \times$ $\left.\left[\tau_{1}, \tau_{2}\right]\right)$, where $0<\tau_{1}<\tau_{2}<\infty$, then $\mathcal{G}, \dot{\mathcal{G}}$, and $\ddot{\mathcal{G}}$ are $L^{\infty}$ since, for example,

$$
|\mathcal{G}|_{\infty} \leq \int_{\mathcal{T}}|g(\cdot, \cdot ; \tau)|_{\infty} \mathrm{d} F(\tau) \leq M_{\infty}
$$

when $\mathcal{T} \subset\left[\tau_{1}, \tau_{2}\right]$. Therefore, $\alpha, \beta$, and $\gamma$ are all also $L^{\infty}$. Thus, for each fixed $F \in \mathcal{P}(\mathcal{T}), \alpha, \beta$, and $\gamma$ satisfy the hypothesis of Theorem 1 in [3, p. 35], so that given $\Phi \in V, \Psi \in H=L^{2}(0,1)$, a unique solution to (6) with (3) exists, and $E \in L^{2}([0, T], V), \dot{E} \in L^{2}([0, T], H)$.

It remains yet to show the continuous dependence of solutions on $F$. First we note that $F_{n} \rightarrow F$ in the Prohorov sense is equivalent to

$$
\int f(\tau) \mathrm{d} F_{n}(\tau) \rightarrow \int f(\tau) \mathrm{d} F(\tau) \quad \forall f \in \mathcal{C}\left[\tau_{1}, \tau_{2}\right] .
$$

Since $\ddot{g}$ is uniformly continuous in $\tau$, we have then that $\alpha\left(t, z ; F_{n}\right) \rightarrow \alpha(t, z ; F)$ a.e. when $F_{n} \rightarrow F$. We will define $\alpha_{n}:=\alpha\left(t, z ; F_{n}\right)$ and $\alpha:=\alpha(t, z ; F)$. Because $\left|\alpha_{n}\right|_{L^{\infty}} \leq M$ for all $n$, the Dominated Convergence Theorem implies that $\alpha_{n} \rightarrow \alpha$ in $L^{2}([0, T] \times[0,1])$. Similarly, we can show that $\beta_{n} \rightarrow \beta$ and $\gamma_{n} \rightarrow \gamma$ in $L^{2}$.

We consider arguments for fixed $\beta$ and $\gamma$. We need to show that $\left(E^{n}, \dot{E}^{n}\right) \rightarrow(E, \dot{E})$ in $L^{2}([0, T], V) \times$ $L^{2}([0, T], H)$ when $\alpha_{n} \rightarrow \alpha$, where $E^{n}$ denotes the solution to (6) corresponding to $\alpha_{n}$. 
We begin by subtracting equation (6) corresponding to $\alpha$ from (6) corresponding to $\alpha_{n}$ to obtain

$$
\begin{aligned}
& \left\langle\ddot{E}^{n}-\ddot{E}, \phi\right\rangle+\left\langle\gamma\left(\dot{E}^{n}-\dot{E}\right), \phi\right\rangle+\left\langle\beta\left(E^{n}-E\right), \phi\right\rangle+\left\langle c^{2}\left(E^{n \prime}-E^{\prime}\right), \phi^{\prime}\right\rangle \\
& \quad+c\left[\dot{E}^{n}(t, 0)-\dot{E}(t, 0)\right] \phi(0)=-\left\langle\int_{0}^{t}\left[\alpha_{n}(t-s, z) E^{n}(s, z)-\alpha(t-s, z) E(s, z)\right] \mathrm{d} s, \phi\right\rangle .
\end{aligned}
$$

By adding and subtracting a mixed term to the right side, we have

$$
\begin{aligned}
& \left\langle\ddot{E}^{n}-\ddot{E}, \phi\right\rangle+\left\langle\gamma\left(\dot{E}^{n}-\dot{E}\right), \phi\right\rangle+\left\langle\beta\left(E^{n}-E\right), \phi\right\rangle+\left\langle c^{2}\left(E^{n \prime}-E^{\prime}\right), \phi^{\prime}\right\rangle \\
& \quad+c\left[\dot{E}^{n}(t, 0)-\dot{E}(t, 0)\right] \phi(0)=-\left\langle\int_{0}^{t} \alpha_{n}\left(E^{n}-E\right) \mathrm{d} s, \phi\right\rangle-\left\langle\int_{0}^{t}\left(\alpha_{n}-\alpha\right) E \mathrm{~d} s, \phi\right\rangle .
\end{aligned}
$$

Following the general procedure in [3] (see also [7,8] for related fundamental ideas and theory), we approximate $E^{n}$ and $E$ in $V_{m}=\operatorname{span}\left\{w_{1}, \ldots, w_{m}\right\}$ by

$$
\begin{aligned}
& E_{m}^{n}(t, z)=\sum_{i=1}^{m} e_{i}^{n}(t) w_{i}(z) \\
& E_{m}(t, z)=\sum_{i=1}^{m} e_{i}(t) w_{i}(z) .
\end{aligned}
$$

Then (8) becomes

$$
\begin{aligned}
& \left\langle\ddot{E}_{m}^{n}-\ddot{E}_{m}, \phi\right\rangle+\left\langle\gamma\left(\dot{E}_{m}^{n}-\dot{E}_{m}\right), \phi\right\rangle+\left\langle\beta\left(E_{m}^{n}-E_{m}\right), \phi\right\rangle+\left\langle c^{2}\left(E_{m}^{n^{\prime}}-E_{m}^{\prime}\right), \phi^{\prime}\right\rangle \\
& \quad+c\left[\dot{E}_{m}^{n}(t, 0)-\dot{E}_{m}(t, 0)\right] \phi(0)=-\left\langle\int_{0}^{t} \alpha_{n}\left(E_{m}^{n}-E_{m}\right) \mathrm{d} s, \phi\right\rangle-\left\langle\int_{0}^{t}\left(\alpha_{n}-\alpha\right) E_{m} \mathrm{~d} s, \phi\right\rangle,
\end{aligned}
$$

which must hold for all $\phi \in V_{m}$. Since $\dot{E}_{m}^{n}$ and $\dot{E}_{m}$ are both in $V_{m}$, we may take $\phi=\dot{E}_{m}^{n}-\dot{E}_{m}$. Then we have

$$
\begin{aligned}
\frac{1}{2} & \frac{\mathrm{d}}{\mathrm{d} t}\left[\left|\dot{E}_{m}^{n}-\dot{E}_{m}\right|_{H}^{2}+\sigma_{1}\left(E_{m}^{n}-E_{m}, E_{m}^{n}-E_{m}\right)\right]+\left|\sqrt{\gamma}\left(\dot{E}_{m}^{n}-\dot{E}_{m}\right)\right|_{H}^{2}+c\left|\dot{E}_{m}^{n}(t, 0)-\dot{E}_{m}(t, 0)\right|^{2} \\
= & \left\langle k\left(E_{m}^{n}-E_{m}\right), \dot{E}_{m}^{n}-\dot{E}_{m}\right\rangle-\left\langle\int_{0}^{t} \alpha_{n}\left(E_{m}^{n}-E_{m}\right) \mathrm{d} s, \dot{E}_{m}^{n}-\dot{E}_{m}\right\rangle \\
& -\left\langle\int_{0}^{t}\left(\alpha_{n}-\alpha\right) E_{m} \mathrm{~d} s, \dot{E}_{m}^{n}-\dot{E}_{m}\right\rangle,
\end{aligned}
$$

where we have used the sesquilinear form $\sigma_{1}: V \times V \rightarrow \mathbb{C}$ defined by

$$
\sigma_{1}(\phi, \psi)=\left\langle c^{2} \phi^{\prime}, \psi^{\prime}\right\rangle_{H}+\langle\hat{\beta} \phi, \psi\rangle_{H}
$$

for $\phi, \psi \in V$ and where $\hat{\beta}=\beta+k>0$ for some sufficiently large $k>0$.

Integration, along with the $V$-ellipticity of $\sigma_{1}$, yields

$$
\begin{aligned}
& \left|\dot{E}_{m}^{n}(t)-\dot{E}_{m}(t)\right|_{H}^{2}+c_{1}\left|E_{m}^{n}(t)-E_{m}(t)\right|_{V}^{2}+2 \int_{0}^{t}\left|\sqrt{\gamma}\left(\dot{E}_{m}^{n}(s)-\dot{E}_{m}(s)\right)\right|_{H}^{2} \mathrm{~d} s \\
& \quad+2 c\left|\dot{E}_{m}^{n}(t, 0)-\dot{E}_{m}(t, 0)\right|_{L^{2}(0, t)}^{2} \leq 2\left|\int_{0}^{t} F_{m}^{n}(\xi) \mathrm{d} \xi\right|
\end{aligned}
$$


where

$$
\begin{aligned}
F_{m}^{n}(\xi)= & \left\langle k\left[E_{m}^{n}(\xi)-E_{m}(\xi)\right], \dot{E}_{m}^{n}(\xi)-\dot{E}_{m}(\xi)\right\rangle-\left\langle\int_{0}^{\xi} \alpha_{n}\left[E_{m}^{n}(s)-E_{m}(s)\right] \mathrm{d} s, \dot{E}_{m}^{n}(\xi)-\dot{E}_{m}(\xi)\right\rangle \\
& -\left\langle\int_{0}^{\xi}\left[\alpha_{n}-\alpha\right] E_{m}(s) \mathrm{d} s, \dot{E}_{m}^{n}(\xi)-\dot{E}_{m}(\xi)\right\rangle \\
= & T_{2}(\xi)+T_{3}(\xi)+T_{4}(\xi) .
\end{aligned}
$$

Following precisely the arguments in [3] we have that

$$
\int_{0}^{t}\left|T_{2}(\xi)\right| \mathrm{d} \xi \leq \int_{0}^{t}\left[\frac{1}{2} k^{2}\left|E_{m}^{n}(\xi)-E_{m}(\xi)\right|_{H}^{2}+\frac{1}{2}\left|\dot{E}_{m}^{n}(\xi)-\dot{E}_{m}(\xi)\right|_{H}^{2}\right] \mathrm{d} \xi
$$

and

$$
\int_{0}^{t}\left|T_{3}(\xi)\right| \mathrm{d} \xi \leq K_{1} \int_{0}^{t}\left|E_{m}^{n}(\xi)-E_{m}(\xi)\right|_{H}^{2} \mathrm{~d} \xi+K_{2} \int_{0}^{t}\left|\dot{E}_{m}^{n}(\xi)-\dot{E}_{m}(\xi)\right|_{H}^{2} \mathrm{~d} \xi
$$

Lastly, we bound the final term by

$$
\begin{aligned}
\left|T_{4}(\xi)\right| & =\left\langle\int_{0}^{\xi}\left[\alpha_{n}(\xi-s)-\alpha(\xi-s)\right] E_{m}(s) \mathrm{d} s, \dot{E}_{m}^{n}(\xi)-\dot{E}_{m}(\xi)\right\rangle \\
& \leq \frac{1}{2}\left|\int_{0}^{\xi}\left[\alpha_{n}(\xi-s)-\alpha(\xi-s)\right] E_{m}(s) \mathrm{d} s\right|_{H}^{2}+\frac{1}{2}\left|\dot{E}_{m}^{n}(\xi)-\dot{E}_{m}(\xi)\right|_{H}^{2} .
\end{aligned}
$$

Since $E_{m}$ is bounded in $C([0, T], V)$,

$$
\begin{aligned}
\left|T_{4}(\xi)\right| & \leq \frac{1}{2}\left(\int_{0}^{\xi} K_{E}\left|\alpha_{n}(\xi-s)-\alpha(\xi-s)\right|_{H} \mathrm{~d} s\right)^{2}+\frac{1}{2}\left|\dot{E}_{m}^{n}(\xi)-\dot{E}_{m}(\xi)\right|_{H}^{2} \\
& \leq \frac{1}{2} K_{E}^{2} t \int_{0}^{\xi}\left|\alpha_{n}(\xi-s)-\alpha(\xi-s)\right|_{H}^{2} \mathrm{~d} s+\frac{1}{2}\left|\dot{E}_{m}^{n}(\xi)-\dot{E}_{m}(\xi)\right|_{H}^{2} .
\end{aligned}
$$

Thus

$$
\int_{0}^{t}\left|T_{4}(\xi)\right| \mathrm{d} \xi \leq \hat{K}_{3} \int_{0}^{t} \int_{0}^{\xi}\left|\alpha_{n}(\xi-s)-\alpha(\xi-s)\right|_{H}^{2} \mathrm{~d} s \mathrm{~d} \xi+K_{4} \int_{0}^{t}\left|\dot{E}_{m}^{n}(\xi)-\dot{E}_{m}(\xi)\right|_{H}^{2} \mathrm{~d} \xi
$$

We observe that

$$
\begin{aligned}
\int_{0}^{t} \int_{0}^{\xi} f(\xi-s) \mathrm{d} s \mathrm{~d} \xi & =\int_{0}^{t} \int_{0}^{t-u} f(u) \mathrm{d} s \mathrm{~d} u \\
& =\int_{0}^{t} f(u)(t-u) \mathrm{d} u \\
& \leq C \int_{0}^{t} f(u) \mathrm{d} u \quad \text { for } t, u \in[0, T]
\end{aligned}
$$

Therefore

$$
\int_{0}^{t}\left|T_{4}(\xi)\right| \mathrm{d} \xi \leq K_{3} \int_{0}^{t}\left|\alpha_{n}(u)-\alpha(u)\right|_{H}^{2} \mathrm{~d} u+K_{4} \int_{0}^{t}\left|\dot{E}_{m}^{n}(\xi)-\dot{E}_{m}(\xi)\right|_{H}^{2} \mathrm{~d} \xi
$$


Using the above bounds, we find that (9) becomes

$$
\begin{aligned}
& \left|\dot{E}_{m}^{n}(t)-\dot{E}_{m}(t)\right|_{H}^{2}+c_{1}\left|E_{m}^{n}(t)-E_{m}(t)\right|_{V}^{2}+2 \int_{0}^{t}\left|\sqrt{\gamma}\left(\dot{E}_{m}^{n}(s)-\dot{E}_{m}(s)\right)\right|_{H}^{2} \mathrm{~d} s \\
& \quad+2 c\left|\dot{E}_{m}^{n}(\cdot, 0)-\dot{E}_{m}(\cdot, 0)\right|_{L^{2}(0, t)}^{2} \\
& \quad \leq C_{1} \int_{0}^{t}\left|E_{m}^{n}(s)-E_{m}(s)\right|_{H}^{2} \mathrm{~d} s+C_{2} \int_{0}^{t}\left|\dot{E}_{m}^{n}(s)-\dot{E}_{m}(s)\right|_{H}^{2} \mathrm{~d} s+C_{3} \int_{0}^{t}\left|\alpha_{n}(s)-\alpha(s)\right|_{H}^{2} \mathrm{~d} s .
\end{aligned}
$$

Noting that $|\cdot|_{H} \leq \mu|\cdot|_{V}$ for some constant $\mu$ and letting

$$
\kappa_{n}:=\int_{0}^{T}\left|\alpha_{n}(s)-\alpha(s)\right|_{H}^{2} \mathrm{~d} s=\left|\alpha_{n}-\alpha\right|_{L^{2}([0, T], H)}^{2},
$$

we then have that (10) implies

$$
\left|\dot{E}_{m}^{n}(t)-\dot{E}_{m}(t)\right|_{H}^{2}+\left|E_{m}^{n}(t)-E_{m}(t)\right|_{V}^{2} \leq v \kappa_{n}+v \int_{0}^{t}\left[\left|E_{m}^{n}(s)-E_{m}(s)\right|_{V}^{2}+\left|\dot{E}_{m}^{n}(s)-\dot{E}_{m}(s)\right|_{H}^{2}\right] \mathrm{d} s
$$

for some $v>0$ independent of $m$ and $n$. Using Gronwall's Inequality we have

$$
\left|\dot{E}_{m}^{n}(t)-\dot{E}_{m}(t)\right|_{H}^{2}+\left|E_{m}^{n}(t)-E_{m}(t)\right|_{V}^{2} \leq v \kappa_{n} \mathrm{e}^{v T} \quad \text { for } t \in[0, T] .
$$

From the existence proof in [3] we have that for each fixed $n, E_{m}^{n} \rightarrow E^{n}$ in $L^{2}([0, T], V)$. Therefore using weak lower semicontinuity of norms we have

$$
\left|E^{n}-E\right|_{L^{2}([0, T], V)}^{2} \leq \liminf _{m}\left|E_{m}^{n}-E_{m}\right|_{L^{2}([0, T], V)}^{2},
$$

with a similar estimate holding for $\dot{E}^{n}-\dot{E}$ in the $L^{2}([0, T], H)$ norm. We may apply these results to the integral of (11) from 0 to $T$ to finally obtain

$$
\left|\dot{E}^{n}-\dot{E}\right|_{L^{2}([0, T], H)}^{2}+\left|E^{n}-E\right|_{L^{2}([0, T], V)}^{2} \leq v \kappa_{n} T \mathrm{e}^{v T} .
$$

Since $\alpha_{n} \rightarrow \alpha$ in $L^{2}$, then $\kappa_{n} \rightarrow 0$, which gives continuous dependence of $(E, \dot{E})$ on $\alpha$. Similar arguments show that $(E, \dot{E})$ depend continuously on $\gamma$ and also on $\beta$. Thus we have that solutions of (6) with (3) depend continuously on the probability measure $F$ in the sense that the map

$$
F \rightarrow(E, \dot{E})
$$

is continuous from $\mathcal{P}(\mathcal{T})$ to $L^{2}([0, T], V) \times L^{2}([0, T], H)$.

Further, this yields that $F \rightarrow J(F)=\sum_{j}\left|E\left(t_{j}, 0 ; F\right)-\hat{E}_{j}\right|^{2}$ is continuous from $\mathcal{P}(\mathcal{T})$ to $\mathbb{R}^{1}$, where $\mathcal{P}(\mathcal{T})$, with the Prohorov metric, is compact for $\mathcal{T}$ compact. Then the general theory of Banks-Bihari in [1] as outlined in [2] can be employed to obtain existence and stability for the inverse problem, as well as an approximation theory which can be used as a basis for a computational methodology.

\section{Conclusion}

We have presented theoretical results on a model for the electric field with multiple electric polarization mechanisms in a dielectric material. This provides a firm foundation for an inverse problem formulation to determine an unknown probability distribution of parameters which describe the dielectric properties of the material. To this end, we have shown the continuous dependence of the solutions with respect to the unknown distributions in the Prohorov metric. This argument, combined with previous 
results on existence and uniqueness in Maxwell systems, demonstrate the well-posedness of the model. Moreover, the theory described in [1,2] can be combined with our results here to provide existence, stability, and an approximation theory for the associated inverse problems.

\section{Acknowledgements}

This research was supported in part by the U.S. Air Force Office of Scientific Research under grant AFOSR F49620-01-1-0026 and in part by the NASA Langley GSRP under grant NGT-1-02004.

\section{References}

[1] H.T. Banks, K.L. Bihari, Modeling and estimating uncertainty in parameter estimation, Inverse Problems 17 (2001) 95-111.

[2] H.T. Banks, D.M. Bortz, G.A. Pinter, L.K. Potter, Modeling and imaging techniques with potential for application in bioterrorism, Technical Report CRSC-TR03-02, Center for Research in Scientific Computation, North Carolina State University, January, 2003, in: H.T. Banks, C. Castillo-Chavez (Eds.), Bioterrorism: Mathematical Modeling Applications in Homeland Security, SIAM Frontiers in Applied Math, vol. FR28, SIAM, Philadelphia, 2003, pp. 129-154 (Chapter 6).

[3] H.T. Banks, M.W. Buksas, T. Lin, Electromagnetic Material Interrogation Using Conductive Interfaces and Acoustic Wavefronts, SIAM Frontiers in Applied Mathematics, Philadelphia, 2000.

[4] P. Billingsley, Convergence of Probability Measures, Wiley, New York, 1968.

[5] M. Davidian, D. Giltinan, Nonlinear Models for Repeated Measurement Data, Chapman and Hall, London, 1998.

[6] R.S. Elliott, Electromagnetics: History, Theory and Applications, IEEE Press, New York, 1993.

[7] J.L. Lions, Optimal Control of Systems Governed by Partial Differential Equations, Springer-Verlag, New York, 1971.

[8] J. Wloka, Partial Differential Equations, Cambridge University Press, 1992. 